\section{Two cases of esophageal eosinophilia: eosinophilic esophagitis or gastro-esophageal reflux disease?}

\author{
Ozlem Yilmaz, ${ }^{1}$ Hacer Ilbilge Ertoy Karagol,1 \\ Erdem Topal,1 Aysel Aksu Unlusoy, ${ }^{2}$ \\ Odul Egritas, 2 Ipek Isik Gonul, 3
}

Arzu Bakirtas1

1Department of Pediatric Allergy and

Asthma, 2Department of Pediatric

Gastroenterology, 3Department

of Pathology, Gazi University School

of Medicine, Ankara, Turkey

\begin{abstract}
Eosinophilic esophagitis (EoE) and gastroesophageal reflux disease are among the major causes of isolated esophageal eosinophilia. Isolated esophageal eosinophilia meeting criteria for EoE may respond to proton pump inhibitor (PPI) treatment. This entity is termed proton pumps inhibitor responsive esophageal eosinophilia (PPI-REE). Gastro-esophageal reflux is thought to comprise a subgroup of patients with PPI-REE. According to the latest guidelines, PPI responsiveness distinguishes people with PPIREE from patients having EoE (non-responders). In this report, two unusual cases with findings belonging to both EoE and PPI-REE are discussed with known and unknown facts.
\end{abstract}

\section{Introduction}

Eosinophilic esophagitis (EoE) is a chronic, immune/antigen-mediated disease characterized clinically by symptoms related to esophageal dysfunction and histologically by eosinophil-predominant inflammation. ${ }^{1}$ EoE is one of the major causes of isolated esophageal eosinophilia. ${ }^{2}$ The other common causes of esophageal eosinophilia including gastroesophageal reflux disease (GERD) may present with similar clinical and histological findings with EoE. There is no clinical or histological finding known to be pathognomonic for EoE. ${ }^{1}$ Isolated esophageal eosinophilia meeting criteria for EoE may respond to proton pump inhibitor (PPI) treatment. This entity is termed proton pump inhibitor responsive esophageal eosinophilia (PPI-REE).1,3 GERD is thought to comprise a subgroup of patients with PPI-REE. According to the latest guidelines, PPI responsiveness distinguishes people with PPI-REE from patients having EoE (non-responders). ${ }^{2}$ In this report, two unusual cases with findings belonging to both EoE and PPI-REE is discussed with known and unknown points.

\section{Case Report \#1}

A six-year-old boy admitted to Pediatric Gastroenterology Clinic with complaints of heartburn along the sternum, regurgitation, nausea, vomiting and epigastric pain with meals. His intermittent symptoms (1-2 times/week) since his third birthday increased in the previous two months. He used alginic acid and domperidone for about a year, but his symptoms did not resolve. On physical examination, his weight was $22.6 \mathrm{~kg}(50-75 \mathrm{p})$ and height was $113 \mathrm{~cm}(50-75 \mathrm{p})$ and all system findings were normal. Serum total IgE was 71.4 $\mathrm{mg} / \mathrm{dL}(0-170 \mathrm{mg} / \mathrm{dL})$, peripheral eosinophil count was $1100 / \mathrm{mm}^{3}$ (10.7\%). An upper gastrointestinal endoscopy revealed felinization in the middle esophagus with normal esophageal mucosa, stomach and duodenum findings. Biopsies obtained from stomach and duodenum were normal, but middle and lower esophageal biopsies (distal: 3 , middle: 4 specimens) revealed 25 and 32 eosinophils per high power field (hpf), respectively and eosinophil microabcesses in the middle esophagus. Barium contrast study of the small intestine was normal. He was consulted to pediatric allergy department for evaluation of atopic diatheses. He had no history of atopic eczema, food allergy and asthma but, described symptoms of intermittent allergic rhinitis (itching and sneezing in the nose). He was skin prick tested with an extensive panel of food and aeroallergens and no sensitization was found. The child was started on $20 \mathrm{mg}$ once daily esomeprozole. The symptoms resolved with this treatment. He was asymptomatic for one year during which time he was lost to follow up, but continued the treatment. Repeat endoscopy was normal macroscopically but, biopsies (distal: 3 , middle: 3 and proximal: 1 specimens) revealed $0 ; 32 ; 0 / \mathrm{hpf}$ in proximal, middle and distal esophagus respectively. He continued to be asymptomatic for one year under PPI treatment. We stopped PPI treatment and wanted to perform a third endoscopy in order to reveal the esophageal pathology again, but the parents did not give consent for a repeat endoscopy. Currently, he is off- treatment and asymptomatic for five months and is being followed for recurrence of symptoms.

\section{Case Report \#2}

An eleven year-old male underwent an upper gastrointestinal endoscopy for dysphagia, drinking excessive liquids during meal times
Correspondence: Ozlem Yilmaz, Department of Pediatric Allergy and Asthma, Gazi University School of Medicine, 06510 Besevler, Ankara, Turkey.

Tel.: +90.312.202.5129 - Fax: +90.312.215.0143.

E-mail: drozlemyilmaz09@gmail.com

Key words: children, eosinophils, esophagitis, proton pumps inhibitors.

Contributions: $\mathrm{OY}, \mathrm{AB}$, had primary responsibility for assessment of cases and prepared the manuscript; HIEK, ET, had responsibility for assessment of cases and revised the manuscript; AUA, OE, performed upper gastrointestinal endoscopies; IIG examined pathological specimens of biopsies.

Conflict of interests: the authors declare no potential conflict of interests.

Received for publication: 6 November 2013.

Revision received: 10 March 2014

Accepted for publication: 11 March 2014.

This work is licensed under a Creative Commons Attribution NonCommercial 3.0 License (CC BYNC 3.0).

○Copyright O. Yilmaz et al., 2014

Licensee PAGEPress, Italy

Pediatric Reports 2014; 6:5160

doi:10.4081/pr.2014.5160

and three episodes of food impaction since five months. Endoscopy showed linear shearing along the esophageal mucosa and trachealization with hyperemia of stomach and duodenal mucosa. Biopsies taken from distal, middle and proximal esophageal mucosa (3, 4 and 5 specimens, respectively) revealed $>30$ eosinophils/hpf. Gastric and duodenal biopsies were normal (Figure 1). The patient was started on $40 \mathrm{mg}$ esomeprozole treatment and was further evaluated in the pediatric allergy department for allergic diseases. His height and weight were $151 \mathrm{~cm} \mathrm{(90} \mathrm{p)} \mathrm{and} 54 \mathrm{~kg}(90$ $97 \mathrm{p})$. The patient had nasal blockade, sneezing, itching of the eyes and nose during spring for four consecutive years (allergic rhinitis) and itching of tongue and throat while eating kiwi and hazelnut (oral allergy syndrome). Serum eosinophil count was $810 / \mathrm{mm}^{3}(10.5 \%)$ and total IgE was $685 \mathrm{mg} / \mathrm{dL}(0-170 \mathrm{mg} / \mathrm{dL})$. Skin prick test was positive to grass $(7 \times 6 \mathrm{~mm})$ and tree pollens $(3 \times 3 \mathrm{~mm})$; cat and dog dander ( $7 \times 8$ and $5 \times 6 \mathrm{~mm})$; hazelnut $(5 \times 5 \mathrm{~mm})$; soy $(3 \times 3 \mathrm{~mm})$ and kiwi $(5 \times 5 \mathrm{~mm})$. Barium contrast study of esophagus, stomach and duodenum were normal. His all symptoms resolved completely with PPI treatment. Repeat endoscopy three months later was normal macroscopically but, esophageal biopsies taken from distal, middle and proximal mucosa revealed 0/20/75 eosinophils/hpf. 


\section{Discussion and Conclusions}

Eosinophilic esophagitis is clinically characterized by symptoms of esophageal dysfunction which varies by age: children most commonly had GERD-like symptoms, whereas adolescents and adults usually present with dysphagia and food impaction. ${ }^{3}$

In this report, we presented two cases with esophageal eosinophilia who had symptoms of esophageal dysfunction. Case \#1 was a younger boy with complains of GERD-like symptoms. Case \#2 was an adolescent having dysphagia and food impaction. Both cases had gross esophageal abnormalities which can be detected in patients with EoE including felinization (transient esophageal rings), trachealization (fixed esophageal rings) and linear shearing of esophageal mucosa during upper gastrointestinal endoscopy. Biopsies revealed isolated eosinophilic infiltration along esophageal mucosa which was denser than the minimum threshold number of eosinophils required for diagnosis of EoE $(\geq 15$ eosinophils/hpf).1,3 According to the proposed diagnostic algorithm, 1,3 both cases were administered high dose PPI treatment which resolved all of the clinical symptoms but, not the entire histology. These diseases cannot be distinguished by the histology. Therefore, PPI treatment is important to discriminate $\mathrm{EoE}$ from PPI-REE. EoE is generally thought to be non-responsive to PPI treatment. The patients were also investigated in detail for compensatory behaviors. Interestingly, more severe complaints of the second case including dysphagia, food impaction and washing food down with liquids recovered completely. Gross histological abnormalities also were not detected in the

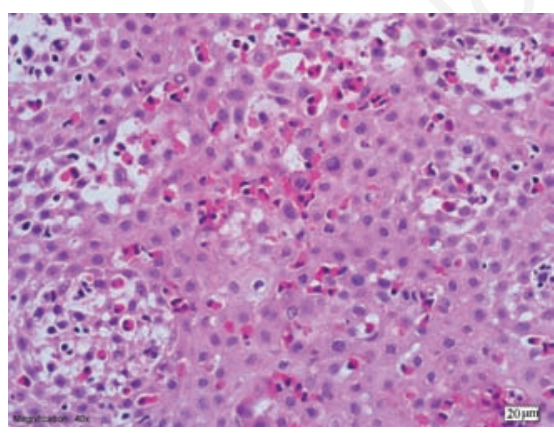

Figure 1. Pretreatment photomicrograph of upper esophageal biopsy of Case \#2. Histological examination of mucosal esophageal biopsy demonstrated numerous intraepithelial eosinophils, eosinophilic microabcesses consistent with eosinophilic esophagitis (Hematoxylin \& Eosin, 400x). second endoscopy after PPI treatment. Another remarkable finding was the dissociation of symptoms with the histology: eosinophilic inflammation persisted in parts of esophagus other than the distal.

According to the latest diagnositc algorithm, patients with isolated esophageal eosinophilia are classified into two groups as PPI-responsive (resolution of both eosinophilia and symptoms) and PPI-nonresponsive (persistence of both eosinophilia and symptoms). The PPI-responsive group is further categorized into GERD (the presence of acid reflux plus esophageal eosinophilia) and non-GERD PPI-REE (acid reflux is not present but, esophageal eosinophilia responds to PPI treatment). Therefore, persistence of eosinophilic infiltration but recovery of symptoms after high dose PPI treatment comprises a diagnostic dilemma according to the latest diagnostic algorithm. ${ }^{2}$ To distinguish EoE from PPI-REE, therapeutic response to PPI or $\mathrm{pH}$ monitoring or both is recommended. It would have been better to decide the presence of GERD if we had performed pHmetry at the initial evaluation of the patients. This is a limitation of our investigations.

In EoE, co-morbid allergic diseases, peripheral eosinophilia and elevated total IgE level were reported in $60 \% ; 5-50 \% ; 70 \%$ percent of cases, respectively. ${ }^{3-6}$ The presented cases resemble to $\mathrm{E} 0 \mathrm{E}$ with the persistence of esophageal eosinophilia (proximal and middle) after PPI treatment, the comorbidity of allergic diathesis and the presence of peripheral eosinophilia and high total IgE levels. However, resolution of eosinophilia in distal esophagus and clinical symptoms with PPI treatment favors the diagnosis of PPI-REE. In other words, the presented cases had features pertaining to both EoE and PPI-REE which was thought as distinct entities. This fact may be explained by the presence of a subgroup also in EoE which is responsive to PPI therapy. The PPI responsiveness of this group may be partial; not enough to ameliorate the eosinophilic infiltration but adequate to normalize the symptomatology. As is the case with many diseases, EoE may be an umbrella term composed of some patient subgroups that may be better understood in the future.

Another possible explanation for the dissociation of the histology and symptoms may be the co-existence of PPI-REE (possibly GERD) and EoE. It is known that acid mediated injury to the mucosa dilates intercellular spaces and favors eosinophilic infiltration. Acid suppression allows time for mucosal healing and prevents further eosinophilic infiltration. ${ }^{7}$ Mucosal healing together with some improvement of eosinophilic infiltration in response to PPI treatment may have caused the abnormalities to decrease below the limit not to cause the symptoms.
In contrast to the persistance of esophageal eosinophilia, the symptoms were resolved in both patients with PPI treatment. In our current knowledge, the long term effects of persistent esophageal inflammation are not known. Therefore, asymptomatic patients were not treated with a specific diatary approach or other therapies such as corticosteroids.

A report by Dohil et al. ${ }^{8}$ showed that initial PPI response may be lost over time. Therefore, clinical follow up of these patients for symptom recurrence is very important and may improve our knowledge about esophageal eosinophilia and eosinophilic esophagitis.

In conclusion, a prudent approach is judicious to the patients with isolated esophageal eosinophilia. Patients with eosinophilic esophagitis may also have a symptomatic response to PPI treatment.

\section{References}

1. Liacouras CA, Furuta GT, Hirano I, et al. Eosinophilic esophagitis: updated consensus recommendations for children and adults. J Allergy Clin Immunol 2011;128:3-20.

2. Dellon ES, Gonsalves N, Hirano I, et al. Clinical guideline: evidenced based approach to the diagnosis and management of esophageal eosinophilia and eosinophilic esophagitis (EoE). Am J Gastroenterol 2013;108:679-92.

3. Furuta GT, Liacouras CA, Collins MH, et al. First international gastrointestinal eosinophil research symposium (FIGERS) subcommittees. Eosinophilic esophagitis in children and adults: a systematic review and consensus recommendations for diagnosis and treatment. Gastroenterology 2007;133:1342-63.

4. Straumann A, Hruz P. What's new in the diagnosis and therapy of eosinophilic esophagitis? Curr Opin Gastroenterol 2009;25:366-71.

5. Blanchard C, Rothenberg ME. Basic pathogenesis of eosinophilic esophagitis. Gastrointest Endosc Clin N Am 2008;18: 133-43.

6. Rothenberg ME. Biology and treatment of eosinophilic esophagitis. Gastroenterology 2009;137:1238-49.

7. Tobey NA, Hosseini SS, Argote CM, et al. Dilated intercellular spaces and shunt permeability in nonerosive acid-damaged esophageal epithelium. Am J Gastroenterol 2004;114:2774-82.

8. Dohil R, Newbury R0, Aceves S. Transient PPI responsive esophageal eosinophilia may be a clinical sub-phenotype of pediatric eosinophilic esophagitis. Dig Dis Sci 2012;57:1413-9. 